Research Paper

\title{
Akt and Hippo Pathways in Ewing's Sarcoma Tumors and Their Prognostic Significance
}

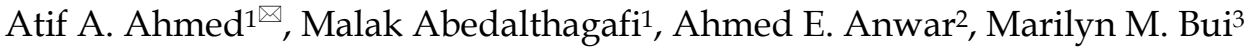 \\ 1. Department of Pathology, King Fahad Medical City, Riyadh, Saudi Arabia \\ 2. Department of Epidemiology and Biostatistics, College of Public Health and Health Informatics, King Saud Bin Abdulaziz University for \\ Health Sciences, Riyadh, Saudi Arabia \\ 3. Department of Anatomic Pathology, Moffitt Cancer Center/University of South Florida, Tampa, Florida, United States
}

$\triangle$ Corresponding author: Atif A. Ahmed, MD, Department of Pathology, Children's Mercy Hospital, Kansas City, MO 64108, United States. Tel: 1-8162343234; Fax: 1-8168021492; Email: aahmed@cmh.edu

๑ 2015 Ivyspring International Publisher. Reproduction is permitted for personal, noncommercial use, provided that the article is in whole, unmodified, and properly cited. See http://ivyspring.com/terms for terms and conditions.

Received: 2015.05.14; Accepted: 2015.07.27; Published: 2015.08.22

\begin{abstract}
Background: Ewing's sarcoma tumor is an aggressive malignancy of bone and soft tissue in children and young adults. Despite advances in modern therapy, metastasis occurs and results in high mortality. Intracellular molecules Yap, Akt, mTOR, and Erk are signaling pathway members that regulate the proliferation of tumor cells.

Objective and Methods: We studied the immunohistochemical expression of these proteins in 36 tumor samples from adult and pediatric patients with Ewing's sarcoma tumors. Patients' age, sex, tumor site, tumor size, clinical stage and survival (overall and disease-free survival) were collected. Tissue microarrays slides were stained with antibodies against Yap, Akt, mTOR, and Erk proteins.

Results: Tumors exhibited variable expression of Yap, Akt, mTOR, and Erk (from negative, low to high), with high levels of expression present in $31 \%, 53 \%, 77 \%$ and $0 \%$ respectively. Immunohistochemical expression of Akt was associated with worse overall and disease-free survival $(p<0.05)$. The other biomarkers did not demonstrate any difference in survival between low versus high expression.

Conclusion: Although Yap, Akt, mTOR, and Erk protein are all expressed in Ewing's sarcoma by immunohistochemistry, only Akt expression is associated with worse prognosis. Larger studies are needed to verify these results and plan targeted therapy, particularly against Akt.
\end{abstract}

Key words: Akt, biomarkers, Ewing's sarcoma, immunohistochemistry, tissue microarray

\section{Introduction}

Ewing sarcoma family tumor (ESFT) is an aggressive sarcoma of bone in children and adults that leads to mortality in substantial number of cases [1-3]. Modern treatment regimens including surgery, chemotherapy and radiotherapy, have improved the outcome of patients with localized disease. However, tumors show a significant metastatic tendency with up to $25 \%$ of patients presenting with metastatic disease. Metastatic tumors are resistant to conventional chemotherapy and radiation resulting in higher mor- tality compared to localized tumors [1-4].

Chromosomal translocations involving the avian erthyroblastosis virus E-twenty-six (ETS) and Ten-eleven translocation (TET) family of proteins are the major oncogenetic events that drive the pathogenesis of ESFT. These genetic events ultimately lead to activation of tyrosine kinases and proliferative pathways that play important roles in tumor proliferation and maintenance. Upregulation of insulin growth factor receptors (IGFR) and subsequent acti- 
vation of the mitogen-activated protein and extracellular signal regulated protein kinases (MAPK-ERK), phosphoinositide 3-kinases (PI3k), and mammalian target of rapamycin (mTor)/Akt pathways have all been identified as major proliferative pathways in ESFT [5-7].

Targeting some of these proliferative molecules by monoclonal inhibitors has resulted in growth inhibition of ESFT tumor cells in vitro models thus giving hopes for development of new therapeutic avenues. However, treatment of ESFT with IGF1R inhibitors has so far yielded less satisfactory results in clinical trials [8] and attention is being given to inhibition of other pathway members as an alternative treatment strategy. Tumor growth control through inhibition of Akt, mTOR and ERK proteins has been demonstrated at the preclinical level suggesting that these inhibitors may be useful as targeted therapy [9-12].

The Hippo pathway is a recently discovered signal transduction pathway whose main components include MST1/2, LATS, Sav1, and YAP. The major function of Hippo signaling pathway is to control cell growth, proliferation, and apoptosis. The yes-associated protein (YAP) is an important transcription co-activator of the Hippo pathway and promotes cell proliferation and epidermal-mesenchymal transition. YAP activation has been linked to tumorigenesis of breast and ovarian cancer $[13,14]$.

Because of tumor heterogeneity, ESFT tumor cells are expected to exhibit variable expression of these proteins and an approach is needed to identify tumors with significant expression of these proteins and any possible association with clinicopathologic prognostic indicators. In this article, we studied the immunohistochemical expression of Yap, Akt, mTOR, and Erk in ESFT tumor specimens with the purpose of correlating that expression with patients' outcome, disease stage, and other clinical data.

\section{Materials and Methods}

\section{Patients:}

This study was carried out in accordance with the research protocol approved by the Institutional Review Boards at the Moffitt Cancer Center and the University of South Florida. Patients' tumor samples were collected from the archives of the Pathology Department over a period of 13 years (1995-2007). Pertinent clinical data including patient's age, sex, tumor site, tumor size, clinical stage and survival (overall and disease-free survival) was compiled (table 1). Patients were clinically monitored up to 14 years after diagnosis. At the end of the follow-up pe- riod, patients were grouped into two categories, as alive or dead. Survival periods were calculated in days from initial diagnosis until death or last known follow-up and were categorized as overall survival (OS) and disease free survival (DFS).

Table 1: Clinicopathological information of patients and data stratification, $\mathrm{N}=36$

\begin{tabular}{lll}
\hline Clinicopathologic parameters & Data stratification & $\mathrm{n}(\%)$ \\
\hline Age (yrs) & $\leq 20$ & $10(27.8)$ \\
& $>20$ & $26(72.2)$ \\
Gender & Males & $23(63.9)$ \\
& Females & $13(36.1)$ \\
Patients' outcome & Alive & $19(52.8)$ \\
& Dead & $17(47.2)$ \\
Tumor site & Primary skeletal & $20(55.5)$ \\
& Primary extraskeletal & $10(27.8)$ \\
Tumor size & Metastatic & $6(16.7)$ \\
& $<10$ cm & $16(44.4)$ \\
& $\geq 10 \mathrm{~cm}$ & $6(16.7)$ \\
Clinical stage & Unknown & $14(38.9)$ \\
& Localized & $12(33.3)$ \\
& Regional extension & $9(25.0)$ \\
& Distant metastasis & $9(25.0)$ \\
& Unknown & $6(16.7)$ \\
\hline
\end{tabular}

\section{Tumor Specimens and Tissue Microarray Preparation:}

Specimens included in the study were from diagnostic biopsies and resection specimens that were collected before and after initial chemotherapy. The diagnoses were confirmed by ancillary studies in all cases; including immunohistochemistry and molecular methods in certain challenging cases. The most representative sections of the tumor and the corresponding portions $(0.6 \mathrm{~mm}$ diameter $)$ of the paraffin-embedded blocks were selected for construction of tissue microarray (TMA) blocks according to a previously described method [15]. Patients with adequate clinical data and follow-up and adequate tumor tissue blocks were enrolled. Two samples were obtained from two representative areas of the tumor and placed adjacent to each other in the TMA tumor.

\section{Immunohistochemistry:}

Phosphorylated signal pathway proteins, $\mathrm{p}$-AKT (Leica Biosystems, Buffalo Grove, IL), p-mTOR (Cell Signaling, Danvers, MA) and p-ERK (Cell Signaling, Danvers, MA), and non-phosphorylated protein Yap-1 (Santa Cruz, Dallas, Texas), were assessed by immunohistochemistry according to the manufacturer's specifications. After optimization of working conditions, automated immunostaining was performed with a Leica Bond Max instrument (Leica, Richmond, Illinois) utilizing a detection system that gives a brown color staining. After antigen retrieval, 
deparaffinized tumor microarray and control slides were sequentially incubated with the primary antibody, a secondary antibody, a polymer conjugate and a coloring reagent. Separate positive controls were made from breast and prostate carcinomas, all of which were previously shown to exhibit expression of the studied proteins. Staining of blood vessels and stromal cells in some tumors served as internal controls. Negative controls were stained similarly except for omission of primary antibodies.

\section{Analysis of Results:}

Stained slides were analyzed by low (x100) and high magnifications $(x 400)$ and compared to negative and positive controls. The extent of immunohistochemical (IHC) expression was scored by two pathologists using the following system: 0 (negative)= no staining; 1+ (low level of expression $)=$ weak staining that is less than that of positive controls and/or staining that is only visible by high magnification; and 2+ (high level of expression) $=$ strong staining that is equal or more than that of positive controls and/or staining that is clearly visible by low magnification. The staining scores of each section of the patient's tumor were averaged into one score.

Kaplan Meier survival curves were also plotted to demonstrate difference in survival between high and low/negative IHC expression groups of all the

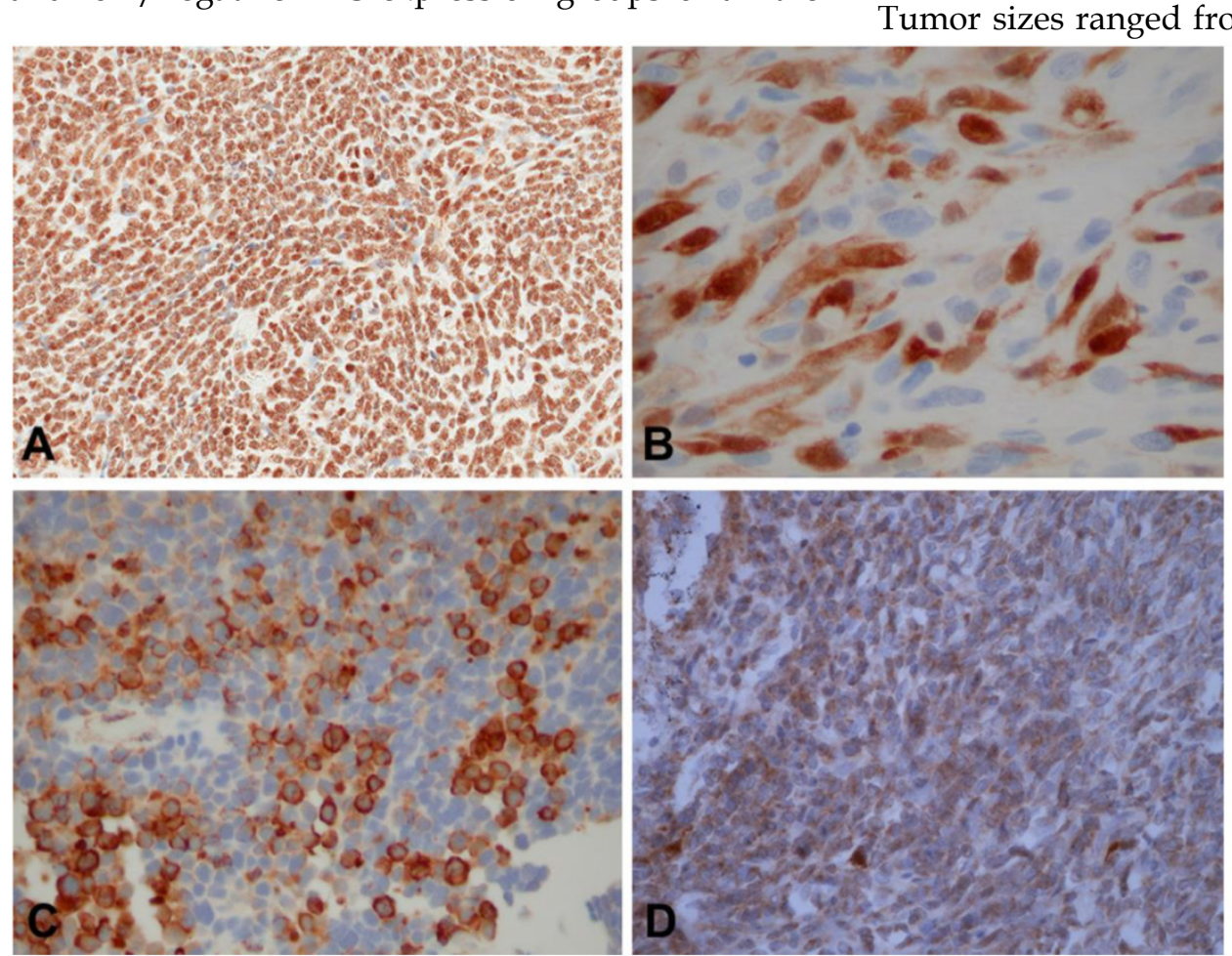

Figure 1: Immunohistochemical staining results of tumor cells. A. Positive immunohistochemical staining of ESFT tumor cells yielded strong nuclear staining for Akt consistent with high expression $(A \times 200)$, cytoplasmic and nuclear staining for YAP $(B \times 400)$, cytoplasmic staining for mTOR $(C \times 200)$, and focal cytoplasmic staining for Erk, consistent with low expression (D $\times 200)$. selected proteins. The effects of clinicopathologic parameters and IHC expression of the studied proteins on overall and disease free survival was also examined by univariate cox proportional hazards model (Chi-squre test).

\section{Results}

\section{Patients:}

Clinical data were collected from 23 male and 13 females patients $(\mathrm{M}: \mathrm{F}=1.8)$ that had an age range of $12-72$ years. Twenty six $(72.2 \%)$ patients were older than 20 years and $10(27.8 \%)$ of the patients were of the pediatric age group ( $\leq 20$ years). Patients had follow-up visits up to 14 years from the primary diagnosis; the shortest follow-up period was 25 days. At the end of the follow-up period, 17(47.2\%) patients were dead and 19 (52.8\%) were alive. Overall survival (OS) ranged from 25 - 5,065 days and DFS from 25 4,224 days. Kaplan-Meier curve and univariate analysis revealed females survived less than males (HR=2.7, 95\% CI: 0.1024-6.987, $\mathrm{p}=0.0445)$.

\section{Tumors:}

Tumor samples were from primary locations in 30 patients and from metastatic sites in six patients. Of the primary locations, $20(55.5 \%)$ patients had skeletal tumors and $10(27.8 \%)$ tumors were extraskeletal. Tumor sizes ranged from $1.2-34.5 \mathrm{~cm}$ (measured in 22 patients). Clinical stage was determined in 30 cases and was grouped into: localized (12 cases), regional extension (9 cases) and distant metastasis (9 cases).

\section{Immunostaining re- sults:}

The staining results are shown in table 2. Positive (low or high expression) staining was identified in $15(46.8 \%)$ of the 32 cases stained for Yap; in 21 $(65.6 \%)$ of the 32 stained for $\mathrm{Akt}$; in $28(90.3 \%)$ of the 31 cases stained for mTor and in $6(19.4 \%)$ of the 31 cases stained with Erk (Figure 1).

For statistical analysis, staining results were grouped into two groups as either negative/low expression (score 0 and $1+$ ) 
versus high expression (score 2+). In Kaplan Meier survival curve, Akt over-expression was associated with worse overall and disease free survival $(p=0.0378$ and $p=0.0471$ respectively, Fig. $2 A$ and $2 B$ ). Univariate cox proportional hazards model confirmed the significant association of Akt expression with worse OS (HR=3.1, 95\% CI: 0.994-9.879, $\mathrm{p}=0.0451)$ and DFS $(\mathrm{HR}=3.0,95 \%$ CI: 0.955-9.499, $\mathrm{p}=0.0459)$ (table 3). The association of Akt expression with OS and DFS was noted more in adult patients ( $>20$ years) than pediatric patients but the difference was not significant $(p>0.05)$. Pediatric patients did not exhibit any association between tumor Akt expression and these bad prognostic indicators. Kaplan Meier survival curves of the remaining biomarkers did not demonstrate differences in survival between tumors with low versus high expression of the studied proteins. No significant association was noted of the positively staining cases and other clinicopathologic parameters such as tumor size, tumor location and clinical stage.

Table 2: Staining results of different antibodies on the TMA slides

\begin{tabular}{llllll}
\hline $\begin{array}{l}\text { Anti- } \\
\text { body }\end{array}$ & $\begin{array}{l}\text { Negative } \\
\text { staining }\end{array}$ & $\begin{array}{l}\text { Low } \\
\text { expres- } \\
\text { sion }\end{array}$ & $\begin{array}{l}\text { High } \\
\text { expres- } \\
\text { sion }\end{array}$ & $\begin{array}{l}\text { Total } \\
\text { stained and } \\
\text { interpreted }\end{array}$ & $\begin{array}{l}\text { Miss- } \\
\text { ing/Unknown } \\
\text { /No inter- } \\
\text { pretable tissue } \\
\text { present }^{*}\end{array}$ \\
\hline Akt & $11(34.4 \%)$ & $4(12.5 \%)$ & $17(53.1 \%)$ & $32(100.0 \%)$ & 4 \\
Yap & $17(53.1 \%)$ & $5(15.6 \%)$ & $10(31.2 \%)$ & $32(100.0 \%)$ & 4 \\
mTor & $3(9.7 \%)$ & $4(12.9 \%)$ & $24(77.4 \%)$ & $31(100.0 \%)$ & 5 \\
Erk & $25(80.6 \%)$ & $6(19.4 \%)$ & $0(0.0 \%)$ & $31(100.0 \%)$ & 5 \\
\hline
\end{tabular}

*Between 4-5 cases were excluded from interpretation because of insufficient viable tumor.

A. Correlation of Akt expression with overall survival (OS)

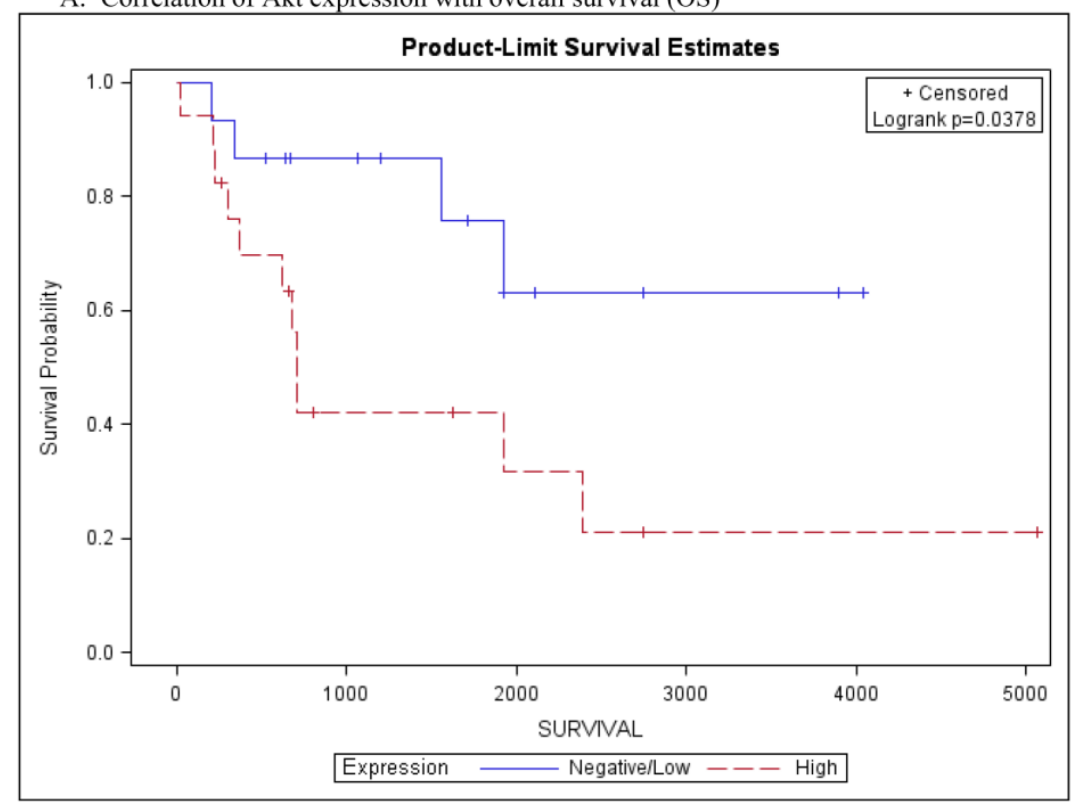

B. Correlation of Akt expression with disease-free survival (DFS)

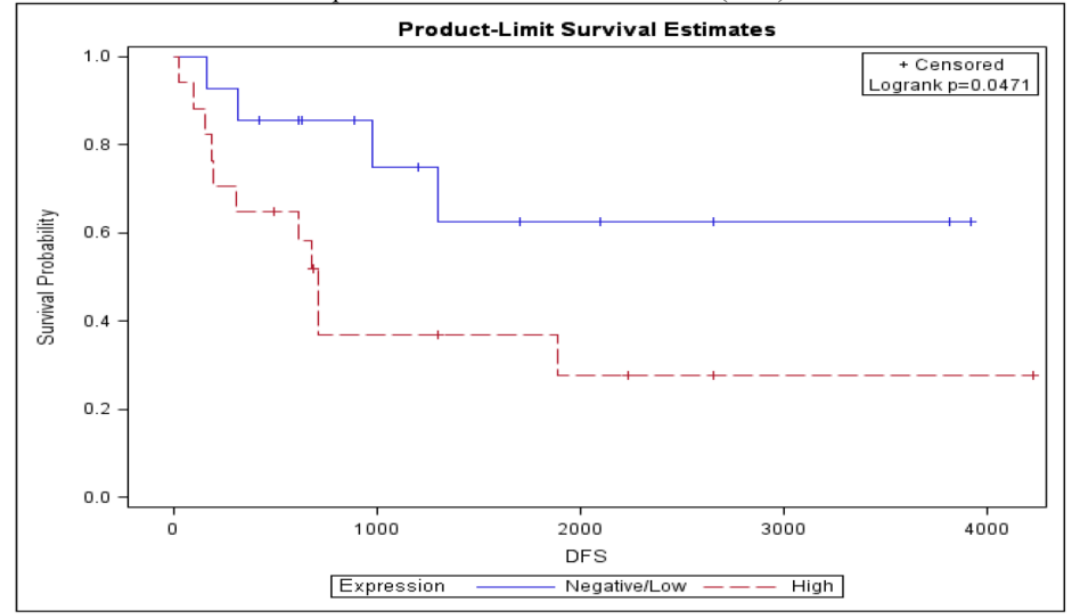

Figure 2: Kaplan-Meier analysis of Akt expression with survival. 
Table 3: Univariate analysis of factors associated with DFS $(\mathrm{N}=36)$

\begin{tabular}{llll}
\hline Characteristics & Levels & HR $(95 \%$ CI for HR $)$ & P-value \\
\hline Sex & Female & $2.7(1.024,6.987)$ & $0.0445^{*}$ \\
Age & Male & 1.0 & - \\
& $>20$ & $0.9(0.324,2.626)$ & 0.8789 \\
Stage & $\leq 20$ & 1.0 & - \\
& Localized & $0.7(0.188,2.263)$ & 0.5012 \\
& Regional extension & $0.9(0.242,3.048)$ & 0.8144 \\
& Distant metastasis & 1.0 & - \\
AKT-Expression & High & $3.0(0.955,9.499)$ & 0.0459 \\
& Negative/Low & 1.0 & - \\
& & & 0.5695 \\
mTOR-Expression & High & $1.4(0.405,5.163)$ & - \\
& Negative/Low & 1.0 & 0.1609 \\
& & & - \\
ERK-Expression & High & $2.3(0.713,7.650)$ & 0.8969 \\
& Negative/Low & 1.0 & - \\
\hline YAP-Expression & High & $0.9(0.295,2.916)$ & \\
& Negative/Low & 1.0 & \\
\hline
\end{tabular}

${ }^{*}$ Chi-square test significant at $\mathrm{a}=0.05$.

\section{Discussion}

This study documents and confirms the presence of activated mTOR/Akt and Yap-Hippo pathways in Ewing's sarcoma family tumors. mTOR and Akt are part of one pathway that is activated subsequent to activation of IGFR and intracellular activation of PI3k [16]. Erk is a member of MAPK/RAF pathway which is connected to the PI3k/Akt/mTOR pathway via multiple points of convergence, cross-talk, and feedback [17]. Expression of these biomarkers in a subset of ESFT patients indicate that they may play roles in tumor growth. The results complement and support findings from in vitro molecular studies previously performed on ESFT cells lines.

Overexpression of Akt was noted in the majority of ESFT tumor cases in this study, consistent with constitutive activation of this molecule in different types of cancer. Although similar findings were mentioned in few other studies $[18,19]$, this study revealed association of Akt over-expression with a worse overall and disease free survival, particularly in adults patients. Akt is a central signaling molecule and is the main cross-talk point in the pathway proliferation of cancers, receiving as well as sending activation signals from and to other molecules. Expression of Akt in worse prognosis ESFT tumors underscores the importance of Akt in the proliferation signaling cascade and the potential benefit of targeted therapy against Akt.

Activation of the Hippo pathway in ESFT is evidenced through overexpression of Yap protein in $47 \%$ of the studied tumor samples. The hippo path- way is connected to Akt and cross-talk exists between the two pathways [20]. Activation of Yap leads to Akt activation. The Yes and Yap proteins are linked to the expression of the polycomb protein BMI, which may play a pivotal role in early ESFT tumorigenesis $[21,22]$. Activation of YAP promotes cell proliferation and transformation and has been linked to tumor progression and worse survival in some malignancies [23].

High expression of mTor is noted in a subset of Ewing's sarcoma patients in our study but did not affect patients' survival, supporting two previous studies $[18,19]$. On the other hand, Erk revealed low levels of expression in this study. Erk activation in ESFT occurs subsequent to chromosomal translocation and different cell lines may show different levels of Erk activation [24]. Lack of association with clinical prognostic parameters suggests a universal role in initial tumor growth subsequent to TET/ETS-induced tumorigenesis.

Mutations of more than one pathway may co-exist in ESFT. Cross-signaling exists between mTor, Akt and MAP/Erk pathways and inhibition of one pathway may still result in the maintenance of tumor growth via secondary mutations and activation of other pathways. The existence of such alternative proliferation mechanisms may be a reason for resistance to targeted inhibition, particularly with the use of single agent inhibitors [25]. Dual or combined targeting of pathways biomarkers may lead to better treatment and clinical outcome. Pathway inhibitors may alternatively be combined with traditional chemotherapy regimens to achieve full therapeutic response [26]. Because of the central role of Akt in ESFT tumorigenesis and its effect on resistance to chemotherapy, a combination targeted therapy including Akt inhibitors may be worth pursuing as a treatment strategy for better tumor control.

In conclusion, Yap, Akt, mTOR, and Erk exhibit variable activation in ESFT. Akt expression may be associated with bad overall and disease-free survival. Although this study is limited by small number of patients and small stained tumor volumes, it is valuable for future directions. Further studies on the role of these proliferation markers, particularly Akt, are necessary for better understanding of their role in targeted therapy.

\section{Acknowledgement}

The authors appreciate the department of pathology, King Fahad Medical City for funding this work and acknowledge the work of the histology laboratory staff of King Fahad Medical City for performing immunohistochemical studies. We would also like to express our gratitude to Dr. Maria Tsokos 
for the critical review of the manuscript and the helpful comments.

\section{Author Contributions}

Atif Ahmed, MD: Conducted experiments, analyzed results and wrote the manuscript

Malak Abedalthagafi, MD: co-reviewed the stained slides and analyzed results.

Ahmed E Anwar, PhD: Conducted statistical analysis

Marilyn Bui, MD, PhD: Provided essential materials and patients data

\section{Conflicts of Interest}

None.

\section{References}

1. Moore DD, Haydon RC. Ewing's sarcoma of bone. Cancer Treat Res. 2014; 162:93-115.

2. Thacker MM, Temple HT, Scully SP. Current treatment for Ewing's sarcoma. Expert rev anticancer ther. 2005; 5:319-31

3. Ludwig JA. Ewing sarcoma: historical perspectives, current state-of-the-art, and opportunities for targeted therapy in the future. Curr Opin Oncol. 2008; 20:412-8

4. Seddon BM, Whelan JS. Emerging chemotherapeutic strategies and the role of treatment stratification in Ewing sarcoma. Paediatr drugs. 2008; 10:93-105

5. Hotfilder M, Sondermann P, Senß A, et al. P13K/AKT is involved in mediating survival signals that rescue Ewing tumor cells from fibroblast growth factor 2-induced cell death. Br J Cancer. 2005; 92: 705-710

6. Chandhanayingyong C, Kim Y, Staples JR, et al. MAP/ERK signaling in osteosarcoma, Ewing sarcoma and chondrosarcomas: Therapeutic implications and future directions. Sarcoma. 2012; 2012: 404810

7. Cironi L, Riggi N, Provero P, et al. IGF1 is a common target gene of Ewing's sarcoma fusion proteins in mesenchymal progenitor cells. PLoS One. 2008; 3: e2634

8. Toretsky JA, Gorlick R. IGF-1R targeted treatment of sarcoma. Lancet Oncol. 2010; 11:105-6

9. Yamamoto T, Ohno T, Wakahara K, et al. Simultaneous inhibition of mitogen-activated protein kinase and phosphatidylinositol 3-kinase pathways augment the sensitivity to actinomycin D in Ewing sarcoma. J Cancer Res Clin Oncol. 2009; 135:1125-36

10. Mateo-Lozano S, Tirado OM, Notario V. Rapamycin induces the fusion-type independent downregulation of the EWS/FLI-1 proteins and inhibits Ewing's sarcoma cell proliferation. Oncogene. 2003; 22:9282-7

11. Kilic-Eren M, Boylu T, Tabor V. Targeting PI3K/Akt represses Hypoxia inducible factor-1alpha activation and sensitizes rhabdomyosarcoma and Ewing's sarcoma cells for apoptosis. Cancer Cell Int. 2013; 13:36

12. Li J, You T, Jing J. MiR-125b inhibits cell biological progression of Ewing's sarcoma by suppressing the PI3K/Akt signalling pathway. Cell Prolif. 2014; 47:152-60

13. Xia Y, Chang T, Wang $\mathrm{Y}$, et al. YAP promotes ovarian cancer cell tumorigenesis and is indicative of a poor prognosis for ovarian cancer patients. PLoS One. 2014; 9:e91770

14. Lamar JM, Stern P, Liu H, et al. The Hippo pathway target, YAP, promotes metastasis through its TEAD-interaction domain. Proc Natl Acad Sci USA. 2012; 109:E2441-50

15. Schlauder SM, Steffensen TS, Morgan M, et al. Assessment of muscarinic and nicotinic acetylcholine receptor expression in primitive neuroectodermal tumor/Ewing family of tumor and desmoplastic small round cell tumor: an immunohistochemical and Western blot study of tissue microarray and cell lines. Fetal Pediatr Pathol. 2008; 27:83-97

16. Scotlandi K, Manara MC, Serra M, et al. Expression of insulin-like growth factor system components in Ewing's sarcoma and their association with survival. Eur J Cancer. 2011; 47:1258-66

17. Saini KS, Loi S, de Azambuja E, et al. Targeting the PI3K/AKT/mTOR and Raf/MEK/ERK pathways in the treatment of breast cancer. Cancer Treat Rev. $2013 ; 39: 935-46$

18. Mora J, Rodríguez E, de Torres C, et al. Activated growth signaling pathway expression in Ewing sarcoma and clinical outcome. Pediatr Blood Cancer. 2012; 58:532-8

19. van de Luijtgaarden AC, Versleijen-Jonkers YM, Roeffen MH, et al. Prognostic and therapeutic relevance of the IGF pathway in Ewing's sarcoma patients. Target Oncol. 2013; 8:253-60

20. Li XJ, Leem SH, Park MH, et al. Regulation of YAP through an Akt-dependent process by 3,3 -diindolylmethane in human colon cancer cells. Int J Oncol. 2013; 43:1992-8
21. Hsu JH, Lawlor ER. BMI-1 suppresses contact inhibition and stabilizes YAP in Ewing sarcoma. Oncogene. 2011; 30:2077-85

22. Douglas D, Hsu JH, Hung L, et al. BMI-1 promotes Ewing's sarcoma tumorigenicity independent of CDKN2A repression. Cancer Res. 2008; 68: 6507-15

23. Wang $\mathrm{H}, \mathrm{Du} Y \mathrm{C}, \mathrm{Zhou} \mathrm{XJ}$, et al. The dual functions of YAP-1 to promote and inhibit cell growth in human malignancy. Cancer Metastasis Rev. 2014; 33:173-81

24. Silvany RE, Eliazer S, Wolff NC, et al. Interference with the constitutive activation of ERK1 and ERK2 impairs EWS/FLI-1-dependent transformation. Oncogene. 2000; 19:4523-30.

25. Chappell WH, Steelman LS, Long JM, et al. Ras/Raf/MEK/ERK and PI3K/PTEN/Akt/mTOR inhibitors: rationale and importance to inhibiting these pathways in human health. Oncotarget. 2011; 2:135-64.

26. Stegeman H, Span PN, Kaanders JH, et al. Improving chemoradiation efficacy by PI3-K/AKT inhibition. Cancer Treat Rev. 2014; 40:1182-1191. 